\title{
Lipophilic fluorescent products of free radicals
}

\author{
Josko Ivica ${ }^{a, b}$, Jiri Wilhelm ${ }^{b}$
}

\begin{abstract}
Background. Fluorescent pigments are the end-products of reactions involving free radical attack on biological molecules and can be formed, for example, in reactions between lipid peroxidation products, mainly unsaturated aldehydes, with free amino groups. Their characteristic emission maximum was found to be at $420-470 \mathrm{~nm}$ after being excited at 340-390 $\mathrm{nm}$. The mechanism of their formation and chemical identity has been revealed in many in vitro studies, in which reactive aldehydes were incubated with amino group-containing molecules. Owing to their intrinsic fluorescent properties and molecular stability these products are easily measured by means of spectrofluorimetry and are used as biomarkers of oxidative stress caused by various triggers. It has been found that the fluorescent products are formed in excess in conditions linked with increased free radical production, such as atherosclerosis, Alzheimer's disease and multiple sclerosis.

Methods. We searched the literature using "MEDLINE" and "Web of Science" in order to get an overview of the state of knowledge about fluorescent products of free radicals, that is, their analysis from in vitro studies, animal and human studies and their use as markers of oxidative damage.

Conclusions. Although their chemical structure may not have been elucidated, the fluorophores formed in this way have found application as markers of oxidative stress in many animal and human studies. In vitro experiments using model reactions have given some clues as to how certain fluorescent pigments arise during oxidative reactions in vivo. Advances in analytical techniques should lead the chemical characterization of pigments of different origin to completeness.
\end{abstract}

Key words: lipofuscin-like pigments, lipid peroxidation, free radicals, fluorescence

Received: February 20, 2012; Accepted with revision: December 6, 2012; Available online: February 13, 2013

http://dx.doi.org/10.5507/bp.2012.112

a Department of Medical Chemistry and Clinical Biochemistry, $2^{\text {nd }}$ Faculty of Medicine, Charles University in Prague and Motol Hospital, Prague, Czech Republic

'Institute of Physiology, Academy of Sciences of Czech Republic, Prague

Corresponding author: Josko Ivica, e-mail:josko.ivica@gmail.com

\section{INTRODUCTION}

Free radicals are chemical species that are, due to unpaired electrons, renowned for their extremely high reactivity. Being indiscriminate with regard what molecule they will react with, in biological environments these reactive species most often attack molecules essential for maintaining cellular homeostasis. Membrane lipids, which contain polyunsaturated fatty acids in their structure, are particularly prone to oxidative damage by free radicals. As a result of free-radical reaction, fatty acids are oxidized into lipid hydroperoxides that, because of their instability, easily break down into products capable of doing further damage to biological material. Finally, relatively stable, i.e. not as reactive as their precursors, chloroform extractable lipophilic fluorescent end-products are formed. Tappel et $\mathrm{al}^{1}$ published the first paper claiming that the lipid peroxidation product - malondialdehyde (MDA), in reaction with free amino groups, produces fluorescent pigments. The end-products were initially called lipofuscin-like pigments (LFP) on the basis of the similarity of their fluorescence properties with those of lipofuscin - the pigment of old age ${ }^{2}$. However, in later studies it was documented that these blue fluorescent compounds were not directly related to yellow-brown fluorescent age pigment - intracellular and extracellular fluorescent material that accumulates in aged tissues. Though other names were coined, like chromolipids $^{4-6}$, advanced lipid peroxidation end-products (ALEs) ( ref. $^{7}$ ) and ceroids, defined as pigments that accumulate due to various pathological processes ${ }^{8,9}$, the original term has survived.

These blue fluorescing pigments have specific excitation and emission maxima. Their emission maximum was found to be at $430-470 \mathrm{~nm}$ on being excited at $260-280 \mathrm{~nm}$ and $350-390 \mathrm{~nm}$, indicating two excitation maxima. As it was reported, the compounds had aminoiminopropene $(-\mathrm{N}=\mathrm{CH}-\mathrm{CH}=\mathrm{CH}-\mathrm{NH}-)$ structure ${ }^{10}$. Another fluorescent group of compounds showing characteristic blue fluorescence was reported by Kikugawa et al. ${ }^{11}$ being derivatives of 1,4-dihydropyridine-3,5-dicarbaldehyde (Fig. 1). These compounds exhibit fluorescence emission maxima at $435-465 \mathrm{~nm}$ when excited at $365-405 \mathrm{~nm}$. Uchida and Itakura $^{12}$ argue the relevance and even contribution of products with aminoenimine structure to the fluorescence of LFP explaining that the fluorophore in these pigments is almost exclusively a dihydropyridine adduct. These fluorescent compounds all originated from reaction between MDA and amino groups of proteins, amino acids or phospholipids. Apart from MDA, another aldehyde produced during lipid peroxidation, 4-hydroxy-2-nonenal 


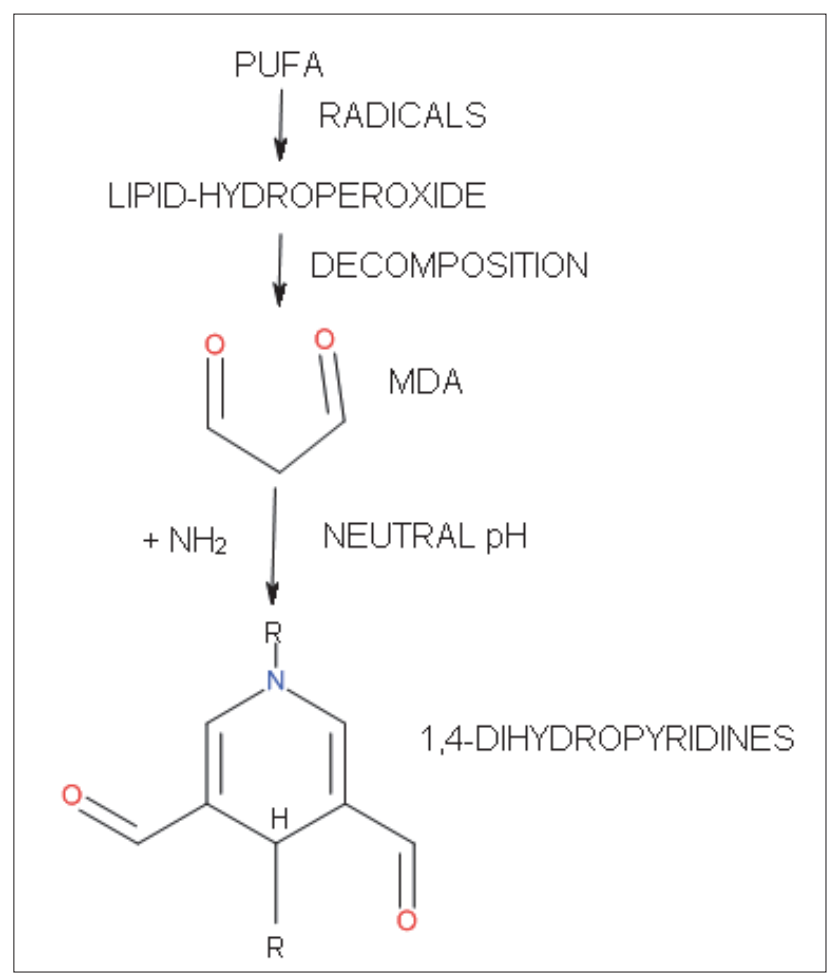

Fig. 1. Scheme of lipid peroxidation and the production of fluorescent end-products.

(4-HNE), contributes to formation of fluorescent LFP $\left(\right.$ ref. $\left.^{6}\right)$. Additionally, numerous fluorescent products have been obtained following reactions between primary amines and lipid peroxidation products other than MDA and 4-HNE (ref. ${ }^{13}$ ).

It appears that LFP are generated in all biological systems. Besides several human and animal studies, they were also detected during plant ageing ${ }^{14-16}$ and even in yeasts ${ }^{17,18}$ and bacteria ${ }^{19}$. LFP were extractable to chloroform from various biological membranes. The original study, and many others, found LFP in cell membranes and mitochondria, but they were also detected in nuclei and nuclear membranes ${ }^{20}$ and microsomes ${ }^{6,21}$.

\section{LIPID PEROXIDATION}

Free radicals and non-radical species of oxygen, collectively known as reactive oxygen species (ROS) are particularly important in biological systems. ROS react with various biological compounds and the consequence of these reactions is most often the damage to those molecules $^{22-26}$.

One group of compounds that are highly sensitive to damage by ROS are polyunsaturated fatty acids (PUFA) found in complex lipids e.g. phospholipids. The process where lipids, in particular PUFA, are oxidatively changed is called lipid peroxidation ${ }^{27,28}$. Lipid peroxidation has been identified as a basic deteriorative process in cellular membranes ${ }^{10}$. Lipid peroxidation is a chain reaction in which a hydrogen atom is abstracted from allylic carbon yielding carbon-centred radical. If this radical reacts with oxygen a peroxyl radical is generated, which can easily react with neighbouring fatty acid forming a lipid-hydroperoxide, thus repeating the cycle ${ }^{29}$.

Further decomposition of lipid-hydroperoxide gives rise to highly reactive aldehydes such as acrolein, MDA, 4-HNE $\left(\right.$ ref. ${ }^{30}$ ) and 4-hydroxyhexena ${ }^{31}$. MDA and 4-HNE are widely used markers of lipid oxidation in a great variety of samples. 4-HNE is formed mainly from linoleic acid, whereas MDA arises mostly as a product of arachidonic acid oxidation ${ }^{32,33}$. The $\alpha, \beta$ - unsaturated aldehydes easily react with many biomolecules (proteins, amino acids, DNA, phospholipids), which results in their impairment. Carbonyl groups of aldehydes react with amino groups of amino acids or phospholipids forming Schiff bases $^{34}$. The products of these reactions are lipofuscin-like fluorophores $1,2,35,36$. The most studied aldehydes participating in fluorescent end-product formation are 4-HNE and MDA (ref. ${ }^{37,38}$ ).

\section{CHARACTERIZATION OF FLUORESCENT END-PRODUCTS}

Fluorophores, produced by cross-linking amino groups with carbonyl moieties of reactive aldehydes, represent a mixture of numerous compounds of known and unknown chemical structure. The fluorescent pigments showing representative lipofuscin-like fluorescence of known structure or at least of known origin have been best characterized and described in experiments where they were formed in vitro by incubating aldehydes derived from lipid oxidation (MDA, 4-HNE) with compounds having a free amino group. Similarly, compounds with a primary amino group can be incubated with PUFA itself or PUFA containing phospholipids in order to analyse arising fluorophores ${ }^{39}$. Fluorescent compounds produced in model reactions in vitro with known and unknown chemical structure have been analysed in order to gain insight into the mechanism of their formation. Trombly and Tappel prepared such fluorescent products in reaction between arachidonic acid and a synthetic phospholipid - dipalmitoyl phosphatidyl etahanolamine (PE), irradiated with UV light to initiate free radical formation, as well as by mixing MDA and PE. They determined excitation maxima of the products, which were at wavelengths $260 \mathrm{~nm}$ and $365 \mathrm{~nm}$, and one emission maximum at $430 \mathrm{~nm}$. The fluorescence observed here lies within the interval typical for lipid peroxidation end-products ${ }^{10}$. In similar in vitro experiments Deng and colleagues synthesized fluorescent products in direct reaction of MDA with biological amines e.g. gamma-aminobutyric acid (GABA) and taurine. In addition, they confirmed the structure of a lipofuscin-like fluorescent product - 1,4-dihydropyridine, which was detected by means of HPLC. Besides this, these findings also show the scavenging properties of various biological amines on reactive aldehydes formed during lipid peroxidation. The amines can attenuate reactive aldehydes' harmful effect as they are able to compete for aldehyde carbonyl groups with amino groups of important macromolecules, thus preventing protein adduct and cross-link formation ${ }^{40,41}$. 
In another experiment, MDA was incubated with pyridoxamine (PM) and this yielded three different products analysed by HPLC and LC-MS: 1-amino-3-iminopropene, 1-pyridoxamino-propenal and a dihydropyridine-pyridinium complex. Dihydropyridine-pyridinium derivative and dihydropyridine are end-products showing typical lipofuscin-like fluorescence. The same authors incubated bovine serum albumin (BSA) with MDA, which resulted in formation of fluorescent products with fluorescence similar to dihydropyridines, and whose intensity was decreased when PM was added, clearly indicating that PM can inhibit, to some extent, the reaction of MDA with proteins ${ }^{42}$.

Slatter et al. found that the principal product of the reaction of MDA and propylamine is a stable dihydropyridine derivative, namely $N$-propyl-4-methyl 2,6-dihydropyridine 3,5-dicarbaldehyde. Likewise, if a protein lysine residue, whose amino group is most reactive towards aldehydes, reacts with MDA the product is $N$-lysyl-4-methyl 2,6-dihydropyridine 3,5-dicarbaldehyde. Additionally, the aldehyde side-chains on the dihydropyridine ring can further react with other amino groups to form protein crosslinks. Similarly, protein amino groups other than those of lysine can directly react with MDA to form adducts ${ }^{33,43}$.

Another reactive aldehyde formed during lipid peroxidation, 4-HNE, is also responsible for production of fluorescent compounds in reactions with amino groups. It has been found that HNE forms a fluorescent hydroxyiminodihydropyrrole derivative with the amino group of lysine residue in oxidized low-density lipoprotein ${ }^{44}$. In another study, where in a model reaction, lysine derivative was exposed to autoxidation of linoleic acid, a fluorophore with hydroxyiminodihydropyrrole structure was produced. The fluorophore was analysed by HPLC with fluorescence detection (360/430 nm excitation/emission). HPLC analysis revealed two different products, one being lysine derivative-HNE adduct, while the second adduct was made of another lipid peroxidation product, 9-hydroxy-12-oxo10-dodecanoic acid, and the lysine derivative. This finding was confirmed by LC-MS analysis ${ }^{45}$. Riazy et al. described lipid peroxidation end-products formed by addition of unfragmented oxidation products of arachidonic and linoleic acid, probably unfragmented aldehydic products, hydroperoxides or endoperoxides, onto amino groups of pro- teins and phospholipids. On analysing these adducts by $\mathrm{MS} / \mathrm{MS}$, the authors found that some of the arachidonic acid-derived lysine-adducts were isolevuglandins containing lactam and hydroxylactam rings. The fluorescence maxima for these products were $360 \mathrm{~nm}$ for excitation and $430 \mathrm{~nm}$ for emission ${ }^{46}$. These findings show that not only decomposition products of lipid-hydroperoxides (mainly aldehydes), but also unfragmented oxidized lipids, can form LFP fluorophores.

\section{METHODOLOGICAL APPROACHES TO LFP ANALYSIS}

To quantify the LFP concentration in a given biological sample, the sample is usually extracted to chloroformmethanol mixture $(2: 1, \mathrm{v} / \mathrm{v})$ which is then washed with water. The chloroform phase is separated and used for fluorescence measurements. This first step in analysis is the most important and potentially flawed by inappropriate ratio of analysed tissue to the volume of the extraction mixture. The intensity of fluorescence is proportional to the concentration of the fluorophores in a reasonable concentration range. However, at high concentrations of the fluorophores proportionality is no longer satisfied, because significant collisional quenching between the molecules of the fluorophore themselves appears. Therefore, if the concentration of LFP fluorophores in the sample is higher than the concentration, above which the fluorescence is no longer proportional to the concentration and can be termed threshold, LFP can produce internal quenching of fluorescence. This concentration, up to which the linear dose-response is obtained, differs in individual samples. As LFP are not chemically characterized, it is not possible to express their concentration in absolute units. LFP in different tissues too have different composition. Thus, the concentration of LFP can be expressed in arbitrary units only and comparison between different tissues is not possible. Generally, if we extract less than $15 \mathrm{mg}$ of tissue in $1 \mathrm{~mL}$ of solvent, we obtain LFP at a concentration that is within linear dose-response range. However, before LFP assay in a new tissue, the range of linear dose-response must be assessed.

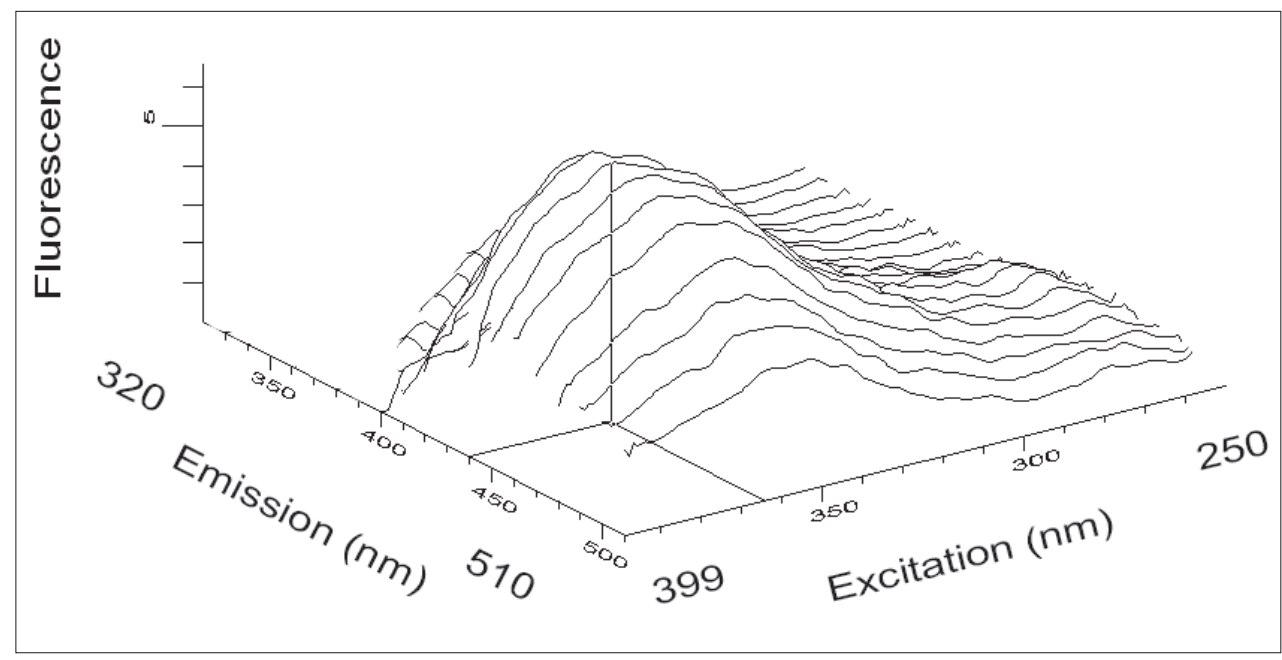

Fig. 2. Fluorescence tridimensional spectral arrays of extracts from isolated mitochondria incubated in vitro with MDA at $37^{\circ} \mathrm{C}$ for 48 hours. Fluorescence intensity is expressed in relative fluorescence units. 


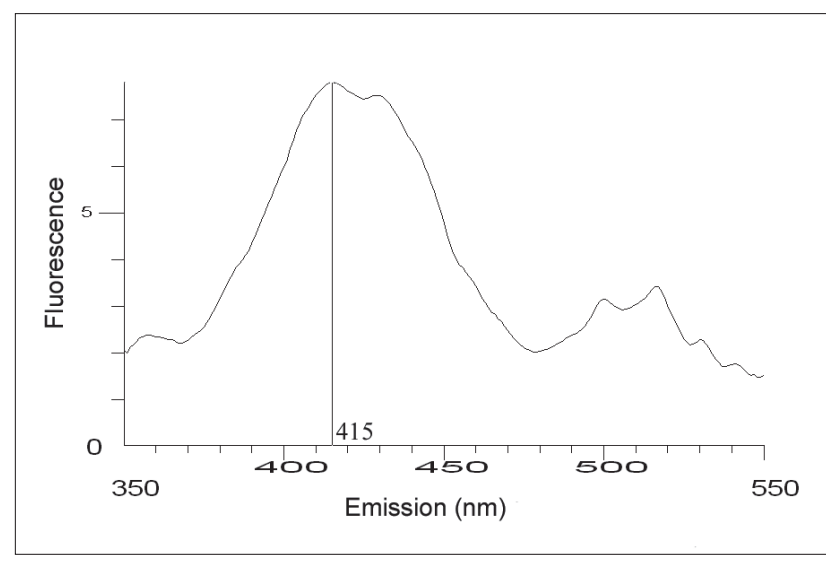

Fig. 3. Fluorescence synchronous spectral arrays of extracts from isolated mitochondria incubated in vitro with MDA at $37^{\circ} \mathrm{C}$ for $48 \mathrm{~h}$. The difference between excitation and emission is $50 \mathrm{~nm}$. Fluorescence intensity is expressed in relative fluorescence units.

The next step usually consists in finding excitation and emission maxima. This is most easily done by measuring 3D spectral arrays of fluorescence spectra. In this way we find even minor fluorescence peaks. For illustration see Fig. 2 which depicts 3D spectral arrays of extracts from isolated mitochondria incubated in vitro with MDA at $37^{\circ} \mathrm{C}$ for $48 \mathrm{~h}$.

LFP is a mixture of several fluorophores that can be characterized by differential synchronous fluorescence spectra. This method has been developed for the analysis of complex mixtures and represents a "fingerprint" of a given sample. This method is especially advantageous if we study changes during certain biological processes (e.g. development of a pathology, or ageing of a cell culture). An example of this spectrum is shown in Fig. 3 (LFP from isolated mitochondria incubated in vitro with MDA at $37{ }^{\circ} \mathrm{C}$ for $48 \mathrm{~h}$ with the constant difference of $50 \mathrm{~nm}$ between excitation and emission wavelengths). Finer resolution of the fluorophores into individual species is obtained by analysing $2^{\text {nd }}$ derivatives of synchronous spectra as seen in Fig. 4 using the same sample as Fig. 2 and 3.

By applying these procedures, we obtain spectroscopically characterized fluorophores that can be quantified in relation to a given standard. We can further resolve the mixture by HPLC with a fluorescence detector. Using HPLC we can resolve spectrally characterized fluorophore into several species. This technique with online mass spectroscopy could lead to final characterization of LFP fluorophores. However, this is a quite complicated task due to the number of fluorescent species and their low concentration. So far this goal has not been achieved.

\section{LFP AS MARKERS OF OXIDATIVE STRESS IN MODEL SYSTEMS}

LFP are currently mainly used as indicators of oxidative stress. As relatively stable end-products of lipid peroxidation, LFP are good markers of free radical production and of consequent damage to lipids. Moreover, they are

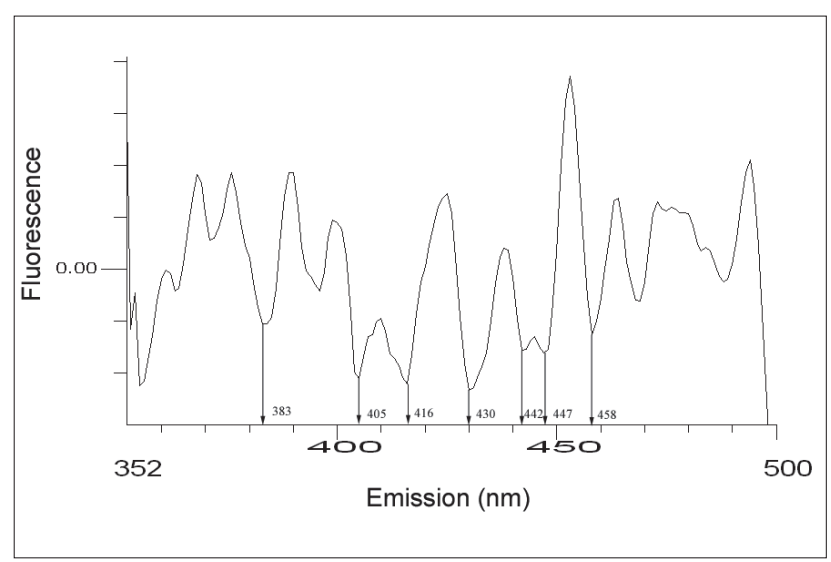

Fig. 4. Second derivatives of synchronous spectral arrays of extracts from isolated mitochondria incubated in vitro with MDA at $37^{\circ} \mathrm{C}$ for $48 \mathrm{~h}$. The vertical arrows indicate the emission maxima of major peaks. Fluorescence intensity is expressed in relative fluorescence units.

used not only as markers of lipid degradation but also to estimate amino acid and protein loss due to cross-linking. So far, LFP have been mostly used as robust markers of oxidative damage without defining the specific chemical identity of compounds representing these pigments. In such cases, the fluorescent pigments are simply markers of free radical production under different circumstances.

Free radical production and oxidative damage in biological systems, caused by various triggers, were assayed by measuring LFP in many studies. Hypoxia, hyperoxia and ischemia/reperfusion are accompanied by increased production of free radicals and LFP were used as markers of oxidative damage ${ }^{36,47-49}$. Elevated LFP concentrations were found in erythrocytes and spleen of rats exposed to hypoxia for different time periods compared to animals that had been kept in normoxic conditions ${ }^{36}$. Measurement of LFP concentration proved to be useful when assessing the antioxidative property of different potential antioxidants $^{49,50}$. Further, LFP were used as indicators of oxidative stress induced by physical activity ${ }^{51}$, phagocytosis of oxidized proteins $\mathrm{s}^{52}$ and ionizing radiation ${ }^{53-60}$. LFP were analysed in various tissues and cell compartments of rats exposed to radiation such as liver homogenates and mitochondria $^{53,56,58}$, skeletal muscles ${ }^{55}$, erythrocytes ${ }^{54}$, sleen $^{54}$ and adipose tissue ${ }^{59,60}$. There are also studies where, for example, erythrocyte ghosts or mitochondria were peroxidized in vitro using different triggers of free radical production (ferrous ions and ascorbic acid) or incubated with lipid peroxidation intermediates (MDA), in order to study the mechanisms of LFP formation or to assess the extent of radical-mediated damage ${ }^{50,61-63}$. Low-density lipoproteins (LDL), which play a very important role in atherosclerotic lesion formation, have been also extensively studied as a model protein, with regard to its oxidation and forming fluorescent adduct on the protein amino groups ${ }^{44,46}$ As mentioned, lipofuscin is the ageing pigment and it accumulates as a normal part of senescence. On the other hand, LFP are produced as a result of oxidative stress, which is connected to pathological processes. LFP are known to be involved in ageing and diseases related to 
it e.g. neurodegenerative and heart diseases. Hence, LFP are a potential marker of oxidative damage to biological material due to increased ROS production in aged subjects. In one such study, LFP were used as a tool to assess the extent of ROS formation in brain cortex of rats during early postnatal development. The highest accumulation was found immediately after birth and the levels were falling up to three months from birth, which is believed to be a period when ageing starts in rats ${ }^{64}$. LFP were also used in an experiment conducted on dogs diagnosed with canine counterpart of senile dementia of Alzheimer's type as one of the markers of lipid peroxidation, which has been proposed to take part in the pathogenesis of this condition ${ }^{65}$. Sen et al. measured fluorescent lipid peroxidation end-products as markers of ageing in rat brains. Aged rat brains had considerably increased accumulation of end-products compared to those from young animals ${ }^{66}$. Davydov and Shvets analysed lipid peroxidation products, with LFP being one of them, in the heart of adult and old rats exposed to stress to assess whether myocardium becomes less resistant against stress at old age ${ }^{67}$. LFP were also measured in brain of rats exposed to lead in order to investigate the possible mechanism of lead toxicity and its role in oxidative stress in brain ${ }^{68}$.

\section{APPLICATION OF LFP ANALYSIS IN BIOMEDICINE}

Since LFP are markers of oxidative damage of lipids and other molecules caused by ROS, their measurement may be a useful tool for monitoring pathological processes linked with oxidative stress. ROS overproduction, which leads to impaired biological molecules, underlies the pathophysiological mechanism of many diseases. The most common disorders, whose developments ROS contribute to, are neurodegenerative diseases e.g. Alzheimer's disease $^{69}$, Parkinson's disease ${ }^{70}$ and multiple sclerosis ${ }^{69}$, atherosclerosis and other vascular diseases ${ }^{25}$, cancer ${ }^{71}$ and diabetes $^{72}$.

The role of ROS in the pathogenesis of Alzheimer's disease $(\mathrm{AD})$ has been widely accepted as there is a strong evidence for it in scientific literature. ROS production exceeding its removal by antioxidant mechanisms leads to oxidative injury in the AD brain. PUFA from brain phospholipids are notably prone to oxidation and there are several studies showing that lipid peroxidation is increased in the AD patient compared with healthy subjects $^{73,74}$. Fluorophores have been described, and identified as HNE-adducts with a pyrrole structure, in the neurons and lesions of AD subjects ${ }^{75}$. Inasmuch as LFP are endproducts of lipid peroxidation, they might be used as specific markers of AD. In a study by Skoumalova and colleagues LFP extracted from red blood cells of patients with $\mathrm{AD}$ and their age-matched controls were analysed in order to find a diagnostic marker easy to measure since blood samples can be routinely taken. They found an increase in LFP formation, measured by means of fluorescence, in patients diagnosed with $\mathrm{AD}$ when compared with controls. As particular fluorophores, obtained by fluorescence measurements, represent a mixture of different compounds they were then resolved into distinctive fractions by HPLC. Chromatograms from these two groups revealed a difference in LFP composition ${ }^{76}$. Another neurodegenerative disease linked with oxidative stress is multiple sclerosis (MS). Yet the relation between oxidative stress and the progression of disability in MS is still unclear. Therefore, Koch and colleagues attempted to assess this relation using LFP as a marker of oxidative stress. LFP was measured in plasma from patients with a different MS disease course and healthy controls. LFP levels were increased in MS patients, compared to controls, but the authors failed to confirm the relation between oxidative stress and disease course and progression ${ }^{77}$.

Vascular diseases characterized by formation of atherosclerotic lesions are associated with oxidative stress. ROS overproduction leads to increased oxidation of low-density lipoprotein, endothelial dysfunction and vascular smooth muscle cell proliferation, which promotes progression of atherosclerotic events ${ }^{78,79}$. Coronary heart disease (CHD) is characterized by accumulation of atherosclerotic plaques and their subsequent rupture in the coronary arteries that supply the myocardium with oxygen and nutrients. Among other parameters that predict disease development and progression, lipid peroxidation products malondialdehyde and fluorescent end-products are ones that are frequently utilized ${ }^{80}$. Wu et al. carried out clinical studies using fluorescent LFP as potential markers of oxidative stress. LFP were measured in plasma from individuals participating in the epidemiological studies to evaluate their ability to predict development of CHD as well as to assess their potential as a global marker of oxidative stress in this type of study. It was found that high levels of fluorescent products were significantly associated with incidence of CHD among individuals without previous cardiovascular events. Further, these markers were found to be an independent risk factor for CHD. The same authors concluded that LFP measured in plasma could be a useful marker of oxidative stress for large epidemiological studies ${ }^{81,82}$. Tertov et al. measured LFP in normal and atherosclerotic areas of the human aorta to deepen our knowledge of atherogenesis and the development of advanced lesions in arterial walls. The level of fluorescent LFP, measured at 360/430 nm (excitation/ emission), did not differ markedly between normal intima and initial lesions. Nevertheless, there was a significant rise in LFP level from atherosclerotic plaques compared to unaffected intima. In addition, it was found that LFP content in lipid extracts from the media underlying normal intima and initial lesions was similar, whereas it was significantly higher in extracts from the media underlying advanced atherosclerotic lesions ${ }^{83}$. Gu et al. stressed the potential usefulness of a specific lipid peroxidation product, omega-(2-carboxyethyl) pyrrole, as a marker for predicting the development of age-related macular degeneration. This protein adduct is formed from a product of free-radical induced oxidation of docosahexaenoic acid ${ }^{84}$. 


\section{CONCLUSION}

LFP are formed as the products of reactions between carbonyls and amino group of biological material. There are many sources of reactive carbonyls. Free radicalmediated peroxidation of lipids from cell membranes contributes substantially to the formation of these very reactive compounds. LFP are a group of compounds that are relatively stable and have intrinsic fluorescence, which makes their analysis easy. Therefore, fluorophores produced in the final stage of the oxidation reactions have great potential as a reliable marker of these processes. To date, these vague fluorophores are still poorly defined and just few of them have been chemically characterized. Nevertheless, LFP have proven to be very useful as indicators of oxidative stress and the pathologies related to it in many animal and some human studies. Improvements in analytical techniques and methods would enable easier characterization and identification of hundreds of fluorophores formed either as consequence or at the onset of various pathological conditions. This is particularly the case with biological samples in which fluorophores, produced as a result of ROS attack on biomolecules, are present in complex mixtures. What complicates understanding of these pigments is that, once formed, they can produce further adducts and cross-links with other molecules. In vitro studies have elucidated some of the mechanisms of LFP formation and have revealed their complete characterization i.e. chemical structure of some fluorophores. This could aid in better understanding the metabolism of all fluorophores formed as products of side reactions in various tissues undergoing pathological processes in vivo.

\section{ABBREVIATIONS}

4-HNE, 4-hydroxy-2-nonenal; AD, Alzheimer's disease; ALEs, Advanced lipid peroxidation end-products; BSA, Bovine serum albumin; CHD, Coronary heart disease; GABA, Gamma-aminobutyric acid; HPLC, High-performance liquid chromatography; LC-MS, liquid chromatography-mass spectrometry; LDL, Lowdensity lipoprotein; LFP, Lipofuscin-like pigments; MDA, Malondialdehyde; MS, Multiple sclerosis; MS/ MS, Tandem mass spectrometry; PE, Dipalmitoyl phosphatidyl etahanolamine; PM, Pyridoxamine; PUFA, Polyunsaturated fatty acid; ROS, Reactive oxygen species; UV, Ultraviolet.

\section{ACKNOWLEDGEMENTS}

This study was supported by grant No. P303/11/0298 of Grant Agency of Czech Republic (GACR).

Authorship contributions: JI, JW: literature search, manuscript writing, study design, data collection, analysis and interpretation; JI: statistical analysis, figures; JW: final approval.

Conflict of interest statement: None declared.

\section{REFERENCES}

1. Chio KS, Tappel AL. Synthesis and characterization of the fluorescent products derived from malonaldehyde and amino acids. Biochemistry 1969;8:2821-6.

2. Chio KS, Reiss U, Fletcher B, Tappel AL. Peroxidation of subcellular organelles: formation of lipofuscinlike fluorescent pigments. Science 1969;166:1535-6.

3. Armstrong D, Wilhelm J, Smid F, Elleder M. Chromatography and spectrofluorometry of brain fluorophores in neuronal ceroid lipofuscinosis (NCL). Mech Ageing Dev 1992;64:293-302.

4. Montfoort A, Bezstarosti K, Groh MM, Koster JF. The influence of the chain length of aldehydes on the fluorescence of chromolipids. FEBS Lett 1987;226:101-4.

5. Koster JF, Slee RG, Van Berkel TJ. On the lipid peroxidation of rat liver hepatocytes, the formation of fluorescent chromolipids and high molecular weight protein. Biochim Biophys Acta 1982;710:230-5.

6. Esterbauer H, Koller E, Slee RG, Koster JF. Possible involvement of the lipid-peroxidation product 4-hydroxynonenal in the formation of fluorescent chromolipids. Biochem J 1986;239:405-9.

7. Seehafer SS, Pearce DA. You say lipofuscin, we say ceroid: defining autofluorescent storage material. Neurobiol Aging 2006;27:576-88.

8. Wang X, Liao Y, Li G, Yin D, Sheng S. A comparative study of artificial ceroid/lipofuscin from different tissue materials of rats. Exp Aging Res. 2008;34:282-95.

9. Yin D. Biochemical basis of lipofuscin, ceroid, and age pigment-like fluorophores. Free Radic Biol Med 1996;21:871-88.

10. Trombly R, Tappel A. Fractionation and analysis of fluorescent products of lipid peroxidation. Lipids 1975;10:441-7.

11. Kikugawa K, Machida Y, Kida M, Kurechi T. Studies on Peroxidized Lipids .3. Fluorescent Pigments Derived from the Reaction of Malonaldehyde and Amino-Acids. Chem Pharm Bull 1981;29:300311.

12. Itakura K, Uchida K. Evidence that malondialdehyde-derived aminoenimine is not a fluorescent age pigment. Chem Res Toxicol 2001;14:473-5.

13. Fukuzawa K, Kishikawa K, Tokumura A, Tsukatani H, Shibuya M. Fluorescent pigments by covalent binding of lipid peroxidation by-products to protein and amino acids. Lipids 1985;20:854-61.

14. Wilhelm J, Wilhelmova N. Accumulation of Lipofuscin-Like Pigments in Chloroplasts from Senescent Leaves of Phaseolus-Vulgaris. Photosynthetica 1981;15:55-60.

15. Wilhelmova N, Domingues PMDN, Srbova M, Fuksova H, Wilhelm J. Changes in nonpolar aldehydes in bean cotyledons during ageing. Biologia Plantarum 2006;50:559-64.

16. Wilhelmova N, Prochazkova D, Machackova I, Vagner M, Srbova $M$, Wilhelm J. The role of cytokinins and ethylene in bean cotyledon senescence. The effect of free radicals. Biologia Plantarum 2004;48:523-9.

17. Wilhelm J, Fuksova H, Schwippelova Z, Vytasek R, Pichova A. The effects of reactive oxygen and nitrogen species during yeast replicative ageing. Biofactors 2006;27:185-93.

18. Munkres KD, Rana RS. Aging of Neurospora-Crassa .7. Accumulation of Fluorescent Pigment (Lipofuscin) and Inhibition of Accumulation by Nordihydroguaiaretic Acid. Mech Ageing Develop 1978;7:399406.

19. Howden PJ, Faux SP. Fibre-induced lipid peroxidation leads to DNA adduct formation in Salmonella typhimurium TA104 and rat lung fibroblasts. Carcinogenesis 1996;17:413-9.

20. Vaca CE, Wilhelm J, Harmsringdahl M. Studies on Lipid-Peroxidation in Rat-Liver Nuclei and Isolated Nuclear-Membranes. Biochim Biophys Acta 1988;958:375-87.

21. Koster JF, Slee RG. Lipid peroxidation of rat liver microsomes. Biochim Biophys Acta. 1980;620:489-99.

22. Halliwell B, Gutteridge JM. Oxygen toxicity, oxygen radicals, transition metals and disease. Biochem J 1984;219:1-14.

23. Pryor WA. Oxy-radicals and related species: their formation, lifetimes, and reactions. Annu Rev Physiol 1986;48:657-67.

24. Bergendi L, Benes L, Durackova Z, Ferencik M. Chemistry, physiology and pathology of free radicals. Life Sci 1999;65:1865-74.

25. Sugamura K, Keaney JF, Jr. Reactive oxygen species in cardiovascular disease. Free Radic Biol Med 2011;51:978-92.

26. Freinbichler W, Colivicchi MA, Stefanini C, Bianchi L, Ballini C, Misini B, Weinberger P, Linert W, Vareslija, D, Tipton KF, Della Corte L. Highly 
reactive oxygen species: detection, formation, and possible functions. Cell Mol Life Sci 2011;68:2067-79.

27. Girotti AW. Mechanisms of lipid peroxidation. J Free Radic Biol Med 1985;1:87-95.

28. van Kuijk FJ, Dratz EA. Detection of phospholipid peroxides in biological samples. Free Radic Biol Med 1987;3:349-54.

29. Reed TT. Lipid peroxidation and neurodegenerative disease. Free Radic Biol Med 2011;51:1302-19.

30. Esterbauer H, Schaur RJ, Zollner H. Chemistry and biochemistry of 4-hydroxynonenal, malonaldehyde and related aldehydes. Free Radic Biol Med 1991;11:81-128.

31. Van Kuijk FJ, Holte LL, Dratz EA. 4-Hydroxyhexenal: a lipid peroxidation product derived from oxidized docosahexaenoic acid. Biochim Biophys Acta 1990;1043:116-8.

32. Esterbauer H, Dieber-Rotheneder M, Waeg G, Striegl G, Jurgens G. Biochemical, structural, and functional properties of oxidized lowdensity lipoprotein. Chem Res Toxicol 1990;3:77-92.

33. Slatter $D A$, Bolton $C H$, Bailey AJ. The importance of lipid-derived malondialdehyde in diabetes mellitus. Diabetologia 2000;43:550-7.

34. Niki E. Lipid peroxidation: physiological levels and dual biological effects. Free Radic Biol Med 2009;47:469-84.

35. Yin D. Studies on age pigments evolving into a new theory of biological aging. Gerontology 1995;41 Suppl 2:159-72.

36. Wilhelm J, Herget J. Hypoxia induces free radical damage to rat erythrocytes and spleen: analysis of the fluorescent end-products of lipid peroxidation. Int J Biochem Cell Biol 1999;31:671-81.

37. Catala A. Lipid peroxidation of membrane phospholipids generates hydroxy-alkenals and oxidized phospholipids active in physiological and/or pathological conditions. Chem Phys Lipids 2009;157:1-11.

38. Del Rio D, Stewart AJ, Pellegrini N. A review of recent studies on malondialdehyde as toxic molecule and biological marker of oxidative stress. Nutr Metab Cardiovasc Dis 2005;15:316-28.

39. Wang JY, Suzuki K, Miyazawa T, Ueki T, Kouyama T. Fluorescence polarization study on the dynamics and location of peroxidized fluorescent phospholipids in liposomes. Arch Biochem Biophys 1996;330:387-94.

40. Deng Y, He NY, Xu LJ, Li XL, Li S, Li ZY, Liu HN. A Rapid Scavenger of the Lipid Peroxidation Product Malondialdehyde: New Perspective of Taurine. Advanced Science Letters 2011;4:442-8.

41. Deng $Y, X u L$, Zeng $X, L i Z$, Qin $B$, He N. New perspective of GABA as an inhibitor of formation of advanced lipoxidation end-products: it's interaction with malondiadehyde. J Biomed Nanotechno 2010;6:318-24

42. Kang Z, Li H, Li G, Yin D. Reaction of pyridoxamine with malondialdehyde: Mechanism of inhibition of formation of advanced lipoxidation end-products. Amino Acids 2006;30:55-61.

43. Slatter DA, Murray M, Bailey AJ. Formation of a dihydropyridine derivative as a potential cross-link derived from malondialdehyde in physiological systems. FEBS Lett 1998;421:180-4.

44. Itakura K, Oya-Ito T, Osawa T, Yamada S, Toyokuni S, Shibata N Kobayashi M, Uchida K. Detection of lipofuscin-like fluorophore in oxidized human low-density lipoprotein. 4-hydroxy-2-nonenal as a potential source of fluorescent chromophore. FEBS Lett 2000:473:249-53.

45. Itakura K, Uchida K. Lysine-derived fluorophores formed by autoxidation of linoleic acid. Chem Phys Lipids 2003;123:187-91.

46. Riazy M, Lougheed M, Adomat HH, Guns ES, Eigendorf GK, Duronio $\mathrm{V}$, Steinbrecher GK. Fluorescent adducts formed by reaction of oxidized unsaturated fatty acids with amines increase macrophage viability. Free Radic Biol Med 2011;51:1926-36.

47. Siskova A, Wilhelm J. The effects of hyperoxia, hypoxia, and ischemia/reperfusion on the activity of cytochrome oxidase from the rat retina. Physiol Res 2001;50:267-73.

48. Wihlmark U, Wrigstad A, Roberg K, Brunk UT, Nilsson SE. Lipofuscin formation in cultured retinal pigment epithelial cells exposed to photoreceptor outer segment material under different oxygen concentrations. Apmis 1996;104:265-71.

49. Ostadalova I, Vobecky M, Chvojkova Z, Mikova D, Hampl V, Wilhelm J, Ostadal B. Selenium protects the immature rat heart against ischemia/reperfusion injury. Mol Cell Biochem 2007;300:259-67.

50. Skoumalova A, Herget J, Wilhelm J. Hypercapnia protects erythrocytes against free radical damage induced by hypoxia in exposed rats. Cell Biochem Funct 2008;26:801-7.

51. Vasankari T, Kujala U, Heinonen O, Kapanen J, Ahotupa M. Measurement of Serum-Lipid Peroxidation during Exercise Using
3 Different Methods - Diene Conjugation, Thiobarbituric Acid Reactive Material and Fluorescent Chromolipids. Clinica Chimica Acta 1995;234:63-9.

52. Shimasaki H, Maeba R, Tachibana R, Ueta N. Lipid peroxidation and ceroid accumulation in macrophages cultured with oxidized low density lipoprotein. Gerontology 1995;41:39-48.

53. Wilhelm J, Sonka J. Time-course of changes in lipofuscin-like pigments in rat liver homogenate and mitochondria after whole body gamma irradiation. Experientia 1981;37:573-4.

54. Wilhelm J, Brzak P, Rejholcova M. Changes of lipofuscin-like pigments in erythrocytes and spleen after whole-body gamma irradiation of rats. Radiat Res 1989;120:227-33.

55. Wilhelm J, Sonka J. Effects of Sublethal Gamma-Irradiation and Exercise on Succinate Oxidase and Metabolites of Lipid Peroxides. 2. Rat Skeletal-Muscle. Agressologie 1980;21:87-91.

56. Wilhelm J, Sonka J. Effect of Sublethal Gamma-Irradiation and Exercise on Succinate Oxidase and Metabolites of Lipid Peroxides. 1. Rat-Liver. Agressologie 1980;21:81-6.

57. Wilhelm J, Sonka J. Modulation of Invivo Metabolic Effects of GammaIrradiation by Thyroid Status Manipulations. Strahlentherapie 1981;157:762-5.

58. Wilhelm J, Sonka J. Ionizing irradiation and fasting in the rat. II. Effect on energy metabolism. Agressologie 1982;23:79-81.

59. Rejholcova M, Wilhelm J. Relationship between lipofuscin-like pigments formation and lipolysis in gamma irradiated rats. Agressologie 1986;27:475-6.

60. Rejholcova M, Wilhelm J. Time course of lipolytic activity and lipid peroxidation after whole-body gamma irradiation of rats. Radiat Res 1989;117:21-5.

61. Wilhelm J, Skoumalova A, Vytasek R, Fisarkova B, Hitka P, Vajner L. Erythrocyte membranes inhibit respiratory burst and protein nitration during phagocytosis by macrophages. Physiol Res 2005;54:5339.

62. Ivica J, Skoumalova A, Topinkova E, Wilhelm J. HPLC Separation of Fluorescent Products of Lipid Peroxidation in Erythrocytes and Mitochondria. Chromatographia 2011;73:S67-S73.

63. Skoumalova A, Madlova P, Topinkova E. End products of lipid peroxidation in erythrocyte membranes in Alzheimer's disease. Cell Biochem Funct 2012;30:205-10.

64. Wilhelm J, Ivica J, Kagan D, Svoboda P. Early postnatal development of rat brain is accompanied by generation of lipofuscin-like pigments. Mol Cell Biochem 2011;347:157-62.

65. Skoumalova A, Rofina J, Schwippelova Z, Gruys E, Wilhelm J. The role of free radicals in canine counterpart of senile dementia of the Alzheimer type. Exp Gerontol 2003;38:711-9.

66. Sen T, Sen N, Jana S, Khan FH, Chatterjee U, Chakrabarti S. Depolarization and cardiolipin depletion in aged rat brain mitochondria: relationship with oxidative stress and electron transport chain activity. Neurochem Int 2007;50:719-25.

67. Davydov VV, Shvets VN. Lipid peroxidation in the heart of adult and old rats during immobilization stress. Exp Gerontol 2001;36:1155-60.

68. Patkova J, Vojtisek M, Tuma J, Vozeh F, Knotkova J, Santorova P Wilhelm J. Evaluation of lipofuscin-like pigments as an index of lead-induced oxidative damage in the brain. Exp Toxicol Pathol 2012;64:51-6.

69. Lassmann H. Mechanisms of neurodegeneration shared between multiple sclerosis and Alzheimer's disease. J Neural Transm 2011;118:747-52.

70. Schapira AH. Oxidative stress in Parkinson's disease. Neuropathol Appl Neurobiol 1995;21:3-9.

71. Klaunig JE, Kamendulis LM, Hocevar BA. Oxidative stress and oxidative damage in carcinogenesis. Toxicol Pathol 2010;38:96-109.

72. Victor VM, Rocha M, Herance R, Hernandez-Mijares A. Oxidative stress and mitochondrial dysfunction in type 2 diabetes. Curr Pharm Des 2011;17:3947-58.

73. McGrath LT, McGleenon BM, Brennan S, McColl D, Mc IS, Passmore AP. Increased oxidative stress in Alzheimer's disease as assessed with 4-hydroxynonenal but not malondialdehyde. Qjm 2001;94:485-90.

74. Montine TJ, Beal MF, Cudkowicz ME, O'Donnell H, Margolin RA, McFarland L, Bachrach AF, Zackert WE, Roberts LJMorrow JD. Increased CSF F2-isoprostane concentration in probable AD. Neurology 1999;52:562-5.

75. Zhu X, Castellani RJ, Moreira PI, Aliev G, Shenk JC, Siedlak SL, Harris PL, Fujioka H, Sayre LM, Szweda PA, Szweda LI, Smith MA, Perry G. Hydroxynonenal-generated crosslinking fluorophore accumulation 
in Alzheimer disease reveals a dichotomy of protein turnover. Free Radic Biol Med 2012;52:699-704.

76. Skoumalova A, Ivica J, Santorova P, Topinkova E, Wilhelm J. The lipid peroxidation products as possible markers of Alzheimer's disease in blood. Exp Gerontol 2011;46:38-42.

77. Koch M, Mostert J, Arutjunyan AV, Stepanov M, Teelken A, Heersema D, De Keyser J. Plasma lipid peroxidation and progression of disability in multiple sclerosis. Eur J Neurol 2007;14:529-33.

78. Madamanchi NR, Runge MS. Mitochondrial dysfunction in atherosclerosis. Circ Res 2007:100:460-73.

79. Puddu P, Puddu GM, Cravero E, De Pascalis S, Muscari A. The emerging role of cardiovascular risk factor-induced mitochondrial dysfunction in atherogenesis. J Biomed Sci. 2009;16:112.

80. Rao V, Kiran R. Evaluation of correlation between oxidative stress and abnormal lipid profile in coronary artery disease. J Cardiovasc Dis Res 2011;2:57-60.
81. Wu T, Rifai N, Willett WC, Rimm EB. Plasma fluorescent oxidation products: independent predictors of coronary heart disease in men. Am J Epidemiol 2007;166:544-51.

82. Wu T, Willett WC, Rifai N, Rimm EB. Plasma fluorescent oxidation products as potential markers of oxidative stress for epidemiologic studies. Am J Epidemiol 2007;166:552-60.

83. Tertov VV, Kaplun VV, Mikhailova IA, Suprun IV, Orekhov AN. The content of lipoperoxidation products in normal and atherosclerotic human aorta. Mol Cell Biochem 2001;225:21-8.

84. Gu X, Meer SG, Miyagi M, Rayborn ME, Hollyfield JG, Crabb JW, Salomon RG. Carboxyethylpyrrole protein adducts and autoantibodies, biomarkers for age-related macular degeneration. J Biol Chem 2003;278:42027-35. 